\title{
Turner syndrome caused by rare complex structural abnormalities involving chromosome $\mathbf{X}$
}

\author{
NIU LI ${ }^{1,2^{*}}$, LI ZHAO $^{3 *}$, JUAN LI $^{3}$, YU DING $^{3}$, YONGNIAN SHEN ${ }^{3}$, \\ XIAODONG HUANG ${ }^{3}$, XIUMIN WANG ${ }^{1,3}$ and JIAN WANG ${ }^{1,2}$ \\ ${ }^{1}$ Department of Medical Genetics; ${ }^{2}$ Institute of Pediatric Translational Medicine; ${ }^{3}$ Department of Internal Medicine, \\ Shanghai Children's Medical Center, Shanghai Jiaotong University School of Medicine, Shanghai 200127, P.R. China
}

Received June 18, 2016; Accepted April 10, 2017

DOI: $10.3892 / \mathrm{etm} .2017 .4756$

\begin{abstract}
Turner syndrome (TS) is a phenotypic heterogeneous genetic disorder caused by the loss of an X-chromosome or $\mathrm{X}$-structural abnormalities in the X-chromosome, and affects approximately 1 in every 2,500 females. The affected individuals may develop diverse clinical features, including short stature, ovarian dysgenesis, skeletal dysplasia, facial abnormalities and other disorders. A constitutional karyotype of 45, $\mathrm{X}$ accounts for nearly $50 \%$ of TS patients, while X-mosaicism and other X-chromosomal structural abnormalities, including deletions, duplications, ring, isodicentric chromosomes, inversions and translocations, have been reported in other cases. The present study reports the results of chromosome microarray analysis (CMA) in two Chinese female TS patients with idiosyncratic karyotypes. The first patient had a karyotype of $46, X, \operatorname{der}(X)$, and the CMA results demonstrated that the derivative chromosome was an abnormal $\mathrm{X}$-chromosome that consisted of three deletions (Xp21.3-p11.23, Xp11.1-q13.1 and $\mathrm{Xq21.31-q28),} \mathrm{as} \mathrm{well} \mathrm{as} \mathrm{three} \mathrm{duplications} \mathrm{(Xp22.33-p21.3,}$ Xp11.23-p11.1 and Xq13.1-q21.31). The karyotype of the second patient was $46, X, \operatorname{der}(X) t(X ; ?)(q$ 22.1;?),inv(11) (q13.5q21), while CMA revealed an Xq21.2-q27.1 duplication and an Xq27.2-q28 deletion. In conclusion, the current study performed genotype-phenotype correlation analysis in two patients and provided novel insight of the genotype of TS.
\end{abstract}

Correspondence to: Dr Xiumin Wang or Dr Jian Wang, Department of Medical Genetics, Shanghai Children's Medical Center, Shanghai Jiaotong University School of Medicine, 1678 Dongfang Road, Shanghai 200127, P.R. China

E-mail: wangxiumin1019@126.com

E-mail: labwangjian@shsmu.edu.cn

*Contributed equally

Key words: Turner syndrome, X-chromosomal structural abnormalities, short stature, short stature homeobox gene

\section{Introduction}

Turner syndrome (TS) is one of the most common chromosomal disorders. It is mainly caused by the partial or complete loss of chromosome $\mathrm{X}$ and affects approximately 1 in every 2,500 females (1). Short stature and ovarian insufficiency are typical features that occur in $>90 \%$ of TS cases. Other features, including skeletal dysplasia (such as cubitus valgus and shortened fourth or fifth metacarpal), heart defects, characteristic facial abnormalities (including low hairline and low-set ears), wide-spaced nipples, nail abnormalities, renal structural anomalies and hearing impairment, have also been reported in TS patients, whereas mental retardation rarely occurs (1-3).

In a previous study in the year of 2004 , approximately $1 / 3$ of patients with TS were diagnosed when they were newborns due to puffy hands and feet or redundant nuchal skin, 1/3 of patients received the diagnosis in mid-childhood because of investigation of short stature and the remaining patients were diagnosed with TS in adolescence for they fail to enter puberty or in adulthood resulting from recurrent pregnancy loss (2). The most important treatment strategy for affected girls is to apply recombinant human growth hormone therapy to improve height and estrogen replacement therapy to improve ovarian function $(2,4)$. Previously results have indicated that, although estrogen treatment may significantly improve nonverbal processing speed, motor performance and verbal and nonverbal memory in patients with TS, the clinical practice is often postponed to puberty, predominantly because estrogen may reduce adult height by accelerating epiphyseal fusion $(5,6)$. A randomized, double-blind, placebo-controlled study suggested that the combination of ultra-low-dose estrogen replacement and growth hormone in childhood not only ameliorated adult height, but also improved the neurocognitive and behavioral function (7).

The clinical features of TS largely depend on the involved regions of the $\mathrm{X}$-chromosome and/or the specific tissues. The most frequent constitutional karyotype of TS patients is 45, $\mathrm{X}$, which accounts for nearly $50 \%$ of the cases and usually manifests as the full TS phenotype. Certain patients with TS are mosaic karyotypes of $45, \mathrm{X}$ or present with both a normal X-chromosome and a structurally complex rearranged X-chromosome. X-structural abnormalities include deletions, duplications, isochromosomes of the long arm, isodicentric 
chromosomes, complex abnormalities with combined deletions and duplications, inversions, rings and translocations. These patients may present certain of the characteristic clinical signs of TS $(8,9)$.

In the present study, the results of cytogenetic analysis and chromosome microarray analysis (CMA) in two girls who presented different features of TS involving two distinct complex $\mathrm{X}$ chromosomal structural rearrangement are reported. Additionally, genotype-phenotype correlation analysis from two patients was performed to provide novel insights into TS.

\section{Materials and methods}

Case 1. The first case reported in the present study involved a 10-year-old girl who was the first child of non-consanguineous healthy parents. The height measurements of both her parents was $170 \mathrm{~cm}$. The patient was born at term via normal delivery, with a normal birth weight of $3,500 \mathrm{~g}$ (reference range: 2,700-3,600 g) and normal length of $51.0 \mathrm{~cm}$ (reference range: $47.7-52.0 \mathrm{~cm}$ ). She was referred to the Shanghai Children's Medical Center (Shanghai, China) for the chief complaint of short stature. Examination indicated an abnormal height of $130.0 \mathrm{~cm}$ (reference range: $131.5-145.1 \mathrm{~cm}$ ), normal weight of $30.0 \mathrm{~kg}$ (reference range: $27.2-47.9 \mathrm{~kg}$ ) and blood pressure of $100 / 70 \mathrm{mmHg}$. X-ray results suggested that the bone age of her phalanges was equivalent to that of a 7 -year-old child. The patient's height had increased by $12.6 \mathrm{~cm}$ in the past 2.5 years and by only $2.2 \mathrm{~cm}$ within 6 months before admission. The patient presented undeveloped bilateral breasts, cubitus valgus, shortened fifth metacarpals, widely-spaced nipples, hypoplastic nails and low hairline. Laboratory examination generated the following results: Follicle-stimulating hormone (FSH), $62.10 \mathrm{mIU} / \mathrm{ml}$ (reference range: $0.65-11.43 \mathrm{mIU} / \mathrm{ml}$ ); luteinizing hormone (LH), $8.73 \mathrm{mIU} / \mathrm{ml}$ (reference range: $0.24-5.66 \mathrm{mIU} / \mathrm{ml}$ ); estradiol, $26.0 \mathrm{pg} / \mathrm{ml}$ (reference range: $20.0-129.3 \mathrm{pg} / \mathrm{ml}$ ); thyrotropin receptor antibody, $50.0 \mathrm{IU} / \mathrm{ml}$ (reference range, $<34.0 \mathrm{IU} / \mathrm{ml}$ ); thyroglobulin antibody, $35.6 \mathrm{IU} / \mathrm{ml}$ (reference range, $<115.0 \mathrm{IU} / \mathrm{ml}$ ); insulin-like growth factor-1 (IGF-1), $151.0 \mathrm{ng} / \mathrm{ml}$ (reference range: $100.0-691.2 \mathrm{ng} / \mathrm{ml}$ ); and IGF-binding protein 3 (IGF-BP3), $4.29 \mathrm{ng} / \mathrm{ml}$ (reference range: $4.33-12.25 \mathrm{ng} / \mathrm{ml}$ ). Magnetic resonance imaging (MRI) and ultrasonography demonstrated an unsatisfactory visualization of bilateral ovaries (streak ovary), while the liver and kidneys were normal. In addition, the MRI examination revealed a small hydrops in the hip joint. Echocardiography and psychological tests revealed no evident abnormalities in the heart and mental development, respectively. The metabolic workup of the patient, including liver transaminases tests, alkaline phosphatase, glucose level, lactate, electrolytes and urinalysis were all normal.

Case 2. The second case investigated in the current study involved a 9-year-old girl who was presented at the Department of Medical Genetics for growth retardation. Her parents were physically healthy and non-consanguineous. No clear signs of mental retardation were detected. The patient was normally delivered at full-term, with a normal birth weight of $3,550 \mathrm{~g}$ and a normal length of $48 \mathrm{~cm}$. The patient was the second childbirth from the second pregnancy, and her older brother did not present any evident abnormalities. Physical examination demonstrated a weight of $21.1 \mathrm{~kg}$ (reference range: $24.1-35.3 \mathrm{~kg}$ ) and a height of $121.5 \mathrm{~cm}$ (reference range: $125.7-138.7 \mathrm{~cm}$ ), with a height increase of only $2.0 \mathrm{~cm}$ reported in the past four months. The patient's heart rhythm was apparently normal, the heart rate was $64 \mathrm{bpm}$ and blood pressure was $84 / 50 \mathrm{mmHg}$. No bilateral breast development was observed, and the bone age of her phalanges was determined to be equivalent to that of a 7-year-old child. In addition, the patient had thick eyebrows, a low hairline and female-appearing genitalia with a normal urethral opening.

Subsequently, the patient underwent a series of additional examinations. The growth hormone-releasing hormone, IGF-1 $(195.0 \mathrm{ng} / \mathrm{ml}$, reference range: $83.6-495.3 \mathrm{ng} / \mathrm{ml})$ and IGF-BP3 $(5.38 \mathrm{ng} / \mathrm{ml}$, reference range: $3.39-11.83 \mathrm{ng} / \mathrm{ml}$ ) levels were in the normal range. FSH $(69.51 \mathrm{mIU} / \mathrm{ml}$, reference range: $0.80-8.41 \mathrm{mIU} / \mathrm{ml})$ and $\mathrm{LH}(9.15 \mathrm{mIU} / \mathrm{ml}$, reference range: $0.05-1.44 \mathrm{mIU} / \mathrm{ml}$ ) levels were markedly higher than the normal range, whereas estradiol level was low $(2.0 \mathrm{pg} / \mathrm{ml}$, reference range: $20.0-74.5 \mathrm{pg} / \mathrm{ml}$ ). Laboratory examination detected no abnormalities in a routine blood and urine tests, electrolyte, insulin and glucose levels, as well as liver, renal and thyroid functions. Ultrasonography indicated streak bilateral ovaries and separated right renal pelvis, and a small uterus with the size of $16 \times 13 \times 9 \mathrm{~mm}$.

Diagnosis and ethical statement. Based on the presented clinical features and laboratory findings, the two subjects were diagnosed with TS according to a guideline from the National Institute of Child Health and Human Development (10). Thus, subsequent cytogenetic analysis and CMA were conducted. The study protocol was approved by the Ethics Committee of the Shanghai Children's Medical Center, and informed consent was obtained from each patient's family.

Cytogenetic analysis. G-banding chromosomal analysis was conducted at the 550-band resolution using peripheral blood samples and standard cell culture techniques as described previously (11). A total of $1.0 \mathrm{ml}$ of peripheral venous blood containing heparin was incubated in a bottle with Roswell Park Memorial Institute (RPMI) 1640 medium (Gibco; Thermo Fisher Scientific, Inc., Waltham, MA, USA) supplemented with $10 \%(\mathrm{v} / \mathrm{v})$ fetal bovine serum (Gibco; Thermo Fisher Scientific, Inc.) and $60 \mathrm{mg} / \mathrm{ml}$ phytohemagglutinin (Gibco; Thermo Fisher Scientific, Inc.) in an atmosphere containing $5 \% \mathrm{CO}_{2}$ incubator at $37^{\circ} \mathrm{C}$ for $72 \mathrm{~h}$ and then three drops of colchicine (Sigma-Aldrich; Merck KGaA; Darmstadt, Germany) was added for $3 \mathrm{~h}$. Cells were centrifuged at $626 \mathrm{x} \mathrm{g} / \mathrm{min}$ for $10 \mathrm{~min}$ at room temperature and the supernatant was discarded. After treatment with $0.075 \mathrm{M} \mathrm{KCl}$ for $30 \mathrm{~min}$ at $37^{\circ} \mathrm{C}$, the cells were fixed in the 3:1 mixture of acetic acid and methanol three times and then 3-4 drops were added on a slide. Slides were placed in an oven for $2 \mathrm{~h}$ at $75^{\circ} \mathrm{C}$ before the cells on the slides were immersed in a preparation of $0.25 \%$ trypsin preparation (Gibco; Thermo Fisher Scientific,Inc.) for $1 \mathrm{sec}$ at $37^{\circ} \mathrm{C}$ and then rinsed in $0.85 \% \mathrm{NaCl}$ at $37^{\circ} \mathrm{C}$ for $1 \mathrm{sec}$. Finally, the slides were stained for $1 \mathrm{~min}$ with Giemsa stain solution (Sigma-Aldrich; Merck KGaA). A total of 20 cells in mitosis metaphase were selected for G-banding karyotype analysis using a light 
Table I. Primers for mmplifying exons and the boundary sequences of the short stature homeobox gene.

\begin{tabular}{lllc}
\hline Exon & \multicolumn{1}{c}{ Forward primer (5'-3') } & \multicolumn{1}{c}{ Reverse primer (5'-3') } & Product size (bp) \\
\hline 1 & & Non-coding & 590 \\
2 & GAGCCCGGAGACCAGTAATT & GAGACGGGAGCTGCAAATG & 578 \\
3 & CAAGAAAACAACGCTCGTGC & TCCAAAAGTCCAGGAACCCC & 579 \\
4 and 5 & AGGTAGTCAGTGATGCAGGG & CTAAGGGCCAGCTGAGAAGT & 668 \\
6 & GAAGGAGGAAAGGCGGGT & ACGGGCAGCCTTGGAGAG & \\
\hline
\end{tabular}

microscope (magnification, $\mathrm{x} 100$ ) in each patient. The karyotype was written according to the International System for Human Cytogenetic Nomenclature 2013 guidelines (12).

CMA. Genomic DNA was extracted from the peripheral blood of the two patients using a QIAamp DNA Blood Mini kit (cat. no. 51106; Qiagen, Hilden, Germany) according to the manufacturer's protocol. Next, genomic hybridization was performed with a CytoScan ${ }^{\mathrm{TM}}$ HD array (Affymetrix; Thermo Fisher Scientific, Inc.), following the manufacturer's instructions. The array was characterized by $>2600,000$ copy number variant (CNV) markers, including 750,000 genotypeable single nucleotide polymorphism probes and $>1900,000$ non-polymorphic probes. Data were visualized and analyzed using the Chromosome Analysis Suite software package version 3.0.0.42 (Affymetrix; Thermo Fisher Scientific, Inc.), with a minimal cutoff of 20 consecutive markers for CNV calling. All reported CNVs were based on the NCBI Human Genome Build 37 database (hg19; National Institutes of Health, Bethesda, MD, USA, https://www.ncbi.nlm.nih .gov/grc/human).

The online mendelian inheritance in man (OMIM) database. The OMIM database (www.omim.org/) was initiated in the early 1960s as a catalog of Mendelian traits and disorders, which is freely available and updated daily. The full-text, referenced overviews in OMIM contain information on all known Mendelian disorders and over 15,000 genes. OMIM focuses on the relationship between phenotype and genotype. OMIM not only links to other genetics resources, but also may be integrated into many databases, such as the UCSC Genome Browser (http://genome.ucsc.edu/), making it easier for us to analyze the data. The OMIM database was used to query the disease-causing gene information, including the location, gene function, inherited model, phenotype and the process of a gene to transform into a pathogenic gene. In the present study, OMIM was use to confirm each gene in the detected CNVs with OMIM.

Sanger sequencing of the (short stature homeobox) SHOX gene. The primers for amplification of the SHOX gene (GenBank accession no. NM_000451) were designed using UCSC ExonPrimer online software (http://genome.ucsc. edu/index.html) and synthesized by Map Biotechnology, Co., Ltd. (Shanghai, China). The primers designed for exon 2 to 6 are listed in Table I. The exons and the exon-intron boundaries were amplified using polymerase chain reaction (PCR). The reaction mixture for each amplification contained
1X Premix Taq (Ex Taq Version 2.0; cat. no. RR003; Takara Biotechnology, Co., Ltd., Dalian, China), 100 ng genomic DNA, and 1 pmol forward and reverse primer in a final volume of $25 \mu \mathrm{l}$. The reaction was performed with the following PCR conditions: Initial denaturation at $95^{\circ} \mathrm{C}$ for $5 \mathrm{~min}$, followed by 19 cycles of $95^{\circ} \mathrm{C}$ for $30 \mathrm{sec}, 65^{\circ} \mathrm{C}$ for $30 \mathrm{sec}, 72^{\circ} \mathrm{C}$ for $45 \mathrm{sec}$, 14 cycles of $95^{\circ} \mathrm{C}$ for $30 \mathrm{sec}, 55^{\circ} \mathrm{C}$ for $30 \mathrm{sec}, 72^{\circ} \mathrm{C}$ for $45 \mathrm{sec}$ and a final elongation step at $72^{\circ} \mathrm{C}$ for 5 min using a $\mathrm{C} 1000^{\mathrm{TM}}$ Thermal Cycler PCR instrument (Bio-Rad Laboratories, Inc., Hercules, CA, USA). The PCR products $(5 \mu \mathrm{l})$ were examined on a $1 \%$ agarose gel and purified using a QIAquick Gel Extraction kit (Qiagen GmbH, Hilden, Germany). The resulting DNA was sequenced using the ABI3730XL sequencer (Applied Biosystems, Thermo Fisher Scientific, Inc.) with the forward and reverse primers. The sequence data were analyzed using Mutation Surveyor ${ }^{\circledR}$ software version 4.0.4 (SoftGenetics, State College, PA, USA).

\section{Results}

Karyotyping results. Karyotyping using chromosomes extracted from peripheral blood is the most commonly employed chromosomal test method for TS (13); therefore, this analysis was performed in the present study. For case 1, G-banding chromosomal analysis revealed a constitutional karyotype of 46,X,der(X), which indicated that an unknown chromosomal fragment replaced the second X-chromosome (Fig. 1A). For case 2, chromosome analysis indicated a karyotype of 46,X,der(X)t(X;?)(q22.1;?),inv(11)(q13.5q21) and, with the exception of one normal X-chromosome, the patient harbored a derivative $\mathrm{X}$-chromosome, which was formed by the translocation of one X-chromosome and an unknown chromosome (Fig. 1B). In addition, a paracentric inversion of chromosome 11 was detected.

CMA results. To ascertain the structure of the marker chromosome, CMA was conducted for the two patients. Three deletions (Xp21.3-p11.23, 2,0186 kb: 27242392-47428395; Xp11.1-q13.1, 10,120 kb: 58,527,154-68,647,456; and Xq21.31-q28, 65,784 kb: 89,449,155-155,233,098), two duplications resulting in triploids (Xp22.33-p21.3, 27,032 kb: 168,551-27,231,894; and Xp11.23-p11.1, 10,902 kb: $47,428,411-58,526,888)$, and one duplication leading to a complex multiploid that included a partial trisomy and a partial tetrasomy (Xq13.1-q21.31, 20,761 kb: 68,678,317-89,439,487) were identified to involve the entire $\mathrm{X}$-chromosome in case 1, as shown in Fig. 2A. The duplicated Xp22.33-p21.3, Xp11.23-p11.1 and Xq13.1-q21.31 contained 97, 45 and 70 

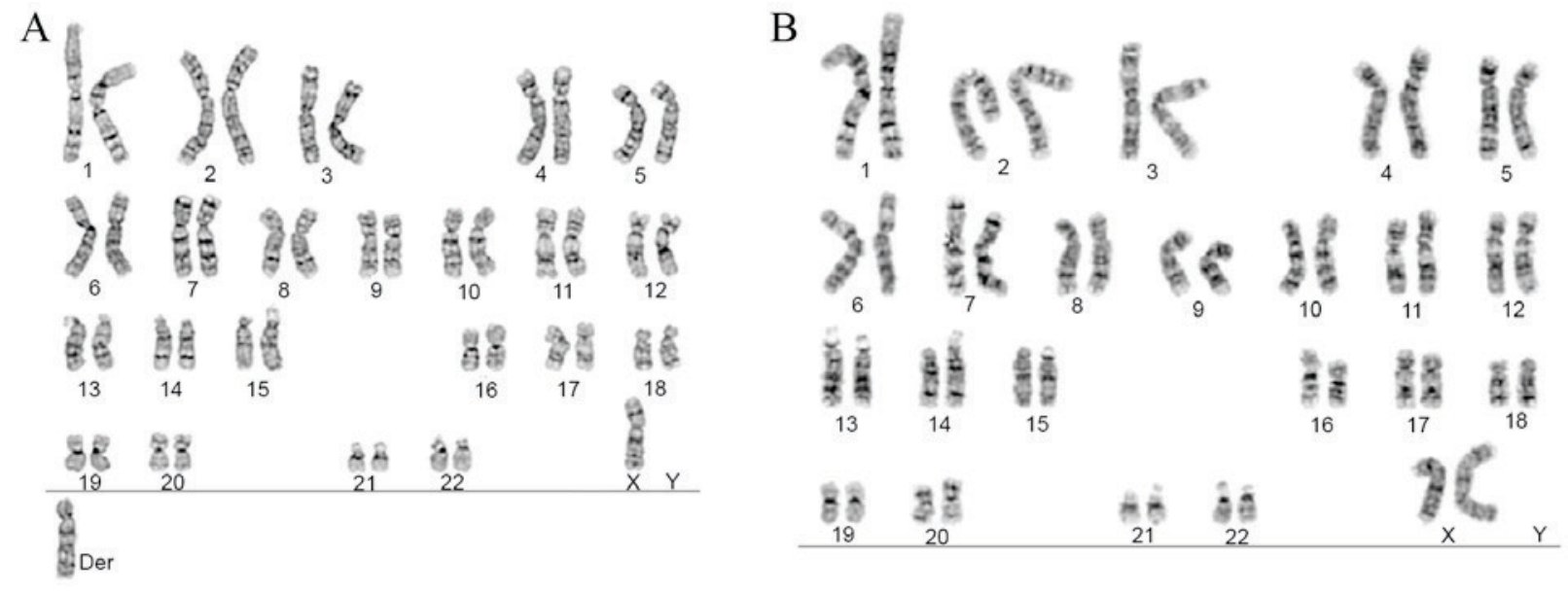

Figure 1. Chromosomal analysis using peripheral blood. (A) Case 1 karyotype was 46,X,der(X). (B) Case 2 karyotype was 46,X,der(X)t(X;?)(q22.1;?),inv(11) (q13.5q21)
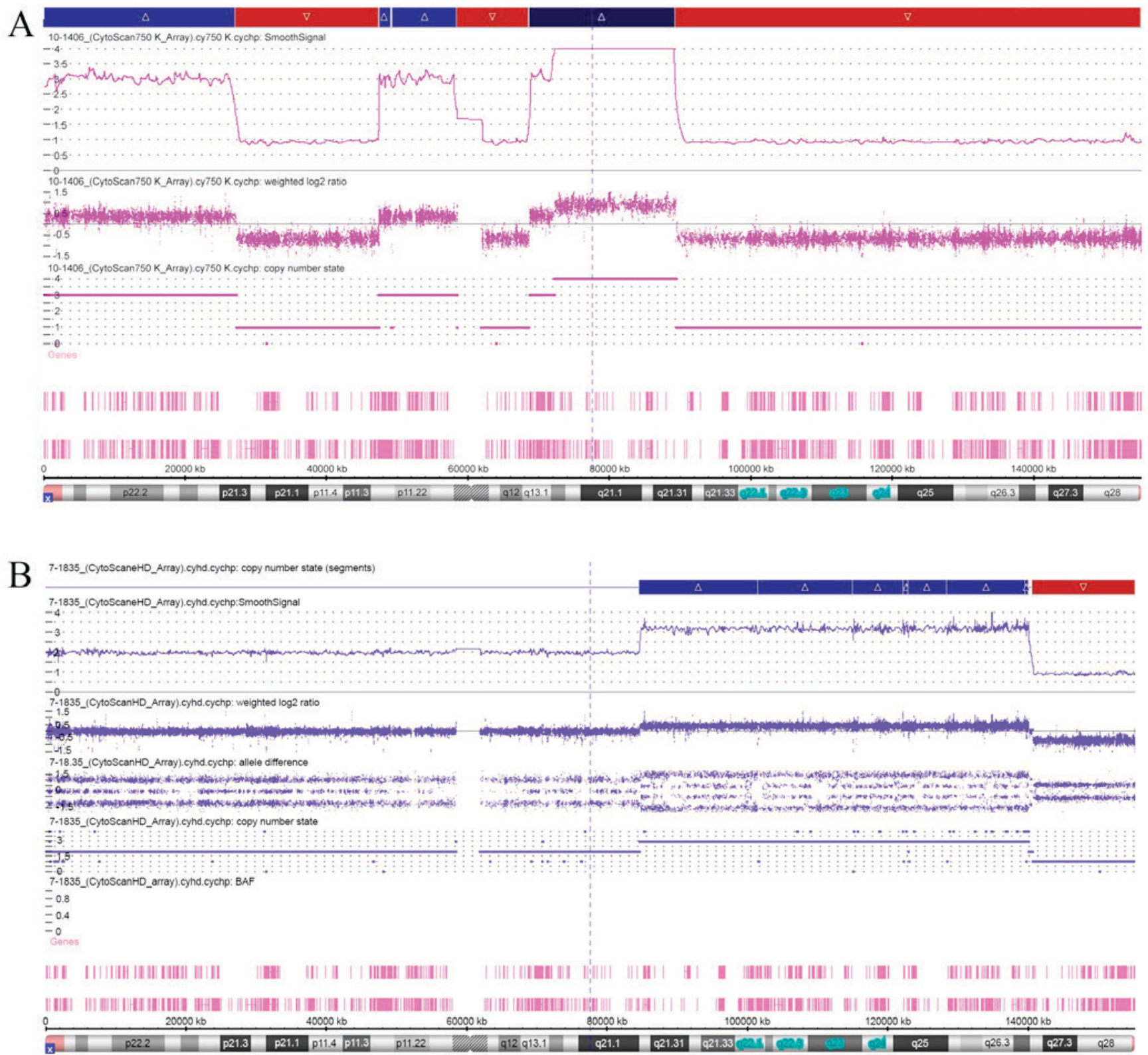

Figure 2. Chromosome microarray analysis of the X-chromosome. (A) Three duplications and three deletions were identified in case 1 . The first two duplications resulted in triploid $\mathrm{X}$, whereas the last duplication consisted of a partial trisomy and a partial tetrasomy. (B) In case 2, one duplication and one deletion were detected in the long arm of the X-chromosome. 
genes, respectively, found in the OMIM catalog of human genes and genetic disorders. The deleted Xp21.3-p11.23, Xp11.1-q13.1 and Xq21.31-q28 contained a total of 54, 15, and 293 OMIM genes, respectively.

Unlike case 1, the CMA results of case 2 revealed two CNVs only in the long arm of X-chromosome: An Xq21.2-q27.1 duplication (triploid, 55,432 kb: 84,647,455-140,079,698), as well as an Xq27.2-q28 deletion (heterozygote, 14,586 kb: $140,647,150-155,233,731)$, as shown in Fig. 2B. The duplicated Xq21.2-q27.1 consisted of 181 OMIM genes, and the deleted Xq27.2-q28 region harbored 112 OMIM genes. No evident clinically significant CNVs were detected in the other chromosomes of the two patients.

Sanger sequencing results. Sanger sequencing was also performed in order to identify point mutations in the SHOX gene of the two patients. The SHOX gene is regarded as the key gene responsible for height increase. However, no mutations were detected in the two TS patients (data not shown).

\section{Discussion}

The present study investigated the genetic variation and clinical features of two female patients with TS. An unclear structural chromosome was identified in the two cases by cytogenetic analysis, followed by CMA. Case 1 presented complex structural abnormalities involving the entire single $\mathrm{X}$-chromosome (deletions in Xp21.3-p11.23, Xp11.1-q13.1 and Xq21.31-q28; and duplications in Xp22.33-p21.3, Xp11.23-p11.1 and Xq13.1-q21.31). In addition, case 1 manifested nearly all phenotypes of TS, including short stature, ovarian insufficiency, smaller uterus, delay in breast development, cubitus valgus, shortened fifth metacarpal, widely-spaced nipples, hypoplastic nails, thyroid disorder and low hairline. Compared with case 1 , the affected region of the X-chromosome in case 2 was limited to the long arm (deletion in Xq27.2-q28 and duplication in Xq21.2-q27.1), and the patient presented with slightly fewer phenotypes, including short stature, ovarian insufficiency, smaller uterus, breast development delay, separated right renal pelvis, thick eyebrows and low hairline.

Deletions of Xp or Xqusually result in TS. Xp deletions are considered to be linked with TS since several genes located on the short arm can escape X-chromosome inactivation, whereas patients with duplications of $\mathrm{Xp}$ or $\mathrm{Xq}$ often present a normal phenotype $(8,14)$. However, compared with case 2 , the phenotypic diversity of case 1 did not fully reflect its complex genotype (particularly involving the short arm), which suggests that TS is highly heterogeneous. Furthermore, the duplications and deletions always appeared in the form of a cross in the two patients, indicating that the duplications compensated for the loss of genetic material that was involved in deletions. To the best of our knowledge, the genotype of case 1 has not previously been reported in the literature, whereas case 2 is partly similar to a previously reported case that presented a karyotype of $46, X, \operatorname{der}(\mathrm{X})$ $\mathrm{t}(\mathrm{X} ; 11)(\mathrm{q} 27.2 ; \mathrm{q} 24.3) \operatorname{dup}(\mathrm{X})(\mathrm{q} 27.1 \mathrm{q} 22.3)(15)$. Array comparative genomic hybridization revealed an Xq27.2-q28 deletion, an 11q24.3-q25 duplication and an inverted duplication of $\mathrm{Xq} 22.3-\mathrm{q} 27.1$ in this previous patient, who presented with primary amenorrhea and mental retardation. Coincidentally, the involved autosome in case 2 of the present study was chromosome 11, where a paracentric inversion was revealed by cytogenetic analysis. No dose modifications of genes on chromosome 11 were observed; however, the effect of this particular inversion requires further investigation.

The SHOX gene, also known as the pseudoautosomal homeobox-containing osteogenic gene, is located at Xp22.33 and Yp11.3, and is involved in skeletal growth and development (16). Mutations or haploinsufficiency of SHOX gene are responsible for approximately two-thirds of the height deficits and skeletal anomalies in TS patients $(17,18)$. In the two cases examined of the current study that presented with short stature, case 1 showed a triploid of the SHOX gene, whereas case 2 harbored the normal number of two alleles. Furthermore, direct sequencing revealed that the two patients did not harbor pathogenic sequence variants in the SHOX gene. Although no direct evidence demonstrated haploinsufficiency of the SHOX gene in the two patients, the role of regulatory regions of SHOX gene require further investigation, where they may be affected by the chromosome abnormalities and lead to gene expression alteration. Additionally, previous studies have suggested a possible role of other regions or genes involved in the control of the stature, such as the Xp11.2-p22.1 region and ITM2A gene $(19,20)$.

Several TS patients with tall stature during puberty have been reported to have resulted from an overdosage of the SHOX gene (21-23). Therefore, follow-up study on whether case 1 undergoes a period of rapid growth during puberty resulting in tall stature would be helpful in clarifying the findings of the present study. Growth hormone $(\mathrm{GH})$ treatment has been demonstrated to be an effective and safe strategy to improve growth in TS patients. Patients treated with $\mathrm{GH}$ were $\sim 5.0 \mathrm{~cm}$ taller as compared with those who received placebo injections, and a greater effect was achieved when combined with estrogen (7). In the present study, the two subjects were offered GH treatment; however, case 1 refused the treatment, whereas case 2 showed a $1.0-\mathrm{cm}$ increase in height after 1 month of treatment.

The thyroid, kidney, hearing and heart of the two cases were also evaluated in the current study. Case 1 harbored an immune dysfunction that involved the thyroid, whereas case 2 had a separated right renal pelvis. No cardiac defects and hearing impairment were observed in the two cases. It is imperative that the patients undergo clinical follow-up and regular examination.

In conclusion, the cases of two TS patients with unusual genotypes were reported in the present study. Furthermore, genotype-phenotype correlation analysis of two patients was performed, providing novel insight regarding the genotype of TS.

\section{Acknowledgements}

The current research was supported by the National Natural Science Foundation of China (grant no. 81472051), the Project of Shanghai Municipal Science and Technology Commission (grant no. 15410722800), and the Project of Shanghai Municipal Education Commission-Gaofeng Clinical Medicine (grant no. 20152529). 


\section{References}

1. Stochholm K, Juul S, Juel K, Naeraa RW and Gravholt CH: Prevalence, incidence, diagnostic delay, and mortality in Turner syndrome. J Clin Endocrinol Metab 91: 3897-3902, 2006.

2. Sybert VP and McCauley E: Turner's syndrome. N Engl J Med 351: 1227-1238, 2004

3. Gonzalez L and Witchel SF: The patient with Turner syndrome: Puberty and medical management concerns. Fertil Steril 98: 780-786, 2012.

4. Ranke MB and Saenger P: Turner's syndrome. Lancet 358: 309-314. 2001

5. Ross JL, Roeltgen D, Feuillan P, Kushner H and Cutler GB Jr: Effects of estrogen on nonverbal processing speed and motor function in girls with Turner's syndrome. J Clin Endocrinol Metab 83: 3198-3204, 1998.

6. Saenger P, Wikland KA, Conway GS, Davenport M, Gravholt CH, Hintz R, Hovatta O, Hultcrantz M, Landin-Wilhelmsen K, Lin A, et al: Recommendations for the diagnosis and management of Turner syndrome. J Clin Endocrinol Metab 86: 3061-3069, 2001.

7. Ross JL, Quigley CA, Cao D, Feuillan P, Kowal K, Chipman JJ and Cutler GB Jr: Growth hormone plus childhood low-dose estrogen in Turner's syndrome. N Engl J Med 364: 1230-1242, 2011.

8. Leppig KA and Disteche CM: Ring X and other structural X chromosome abnormalities: $\mathrm{X}$ inactivation and phenotype. Semin Reprod Med 19: 147-157, 2001

9. Ogata $\mathrm{T}$ and Matsuo N: Turner syndrome and female sex chromosome aberrations: Deduction of the principal factors involved in the development of clinical features. Hum Genet 95: 607-29, 1995.

10. Bondy CA; Turner Syndrome Study Group: Care of girls and women with Turner syndrome: A guideline of the turner syndrome study group. J Clin Endocrinol Metab 92: 10-25, 2007.

11. Seabright M: A rapid banding technique for human chromosomes. Lancet 2: 971-972, 1971.

12. Schaffer LG, McGowan-Jordan J and Schmid M: ISCN 2013: An International System for Human Cytogenetic Nomenclature Basel: Karger, 2013.

13. Wolff DJ, Van Dyke DL and Powell CM; Working Group of the ACMG Laboratory Quality Assurance Committee: Laboratory guideline for Turner syndrome. Genet Med 12: 52-55, 2010.
14. Carrel L, Cottle AA, Goglin KC and Willard HF: A first-generation X-inactivation profile of the human X chromosome. Proc Natl Acad Sci USA 96: 14440-14444, 1999.

15. Chen CP, Lin SP, Chern SR, Kuo YL, Wu PS, Chen YT, Lee MS and Wang W: Array CGH characterization of an unbalanced $\mathrm{X}$-autosome translocation associated with Xq27.2-qter deletion, 11q24.3-qter duplication and Xq22.3-q27.1 duplication in a girl with primary amenorrhea and mental retardation. Gene 535 88-92, 2014.

16. Boucher CA, Sargent CA, Ogata T and Affara NA: Breakpoint analysis of Turner patients with partial Xp deletions: Implications for the lymphoedema gene location. J Med Genet 38: 591-598, 2001.

17. Clement-Jones M, Schiller S, Rao E, Blaschke RJ, Zuniga A, Zeller R, Robson SC, Binder G, Glass I, Strachan T, et al: The short stature homeobox gene SHOX is involved in skeletal abnormalities in Turner syndrome. Hum Mol Genet 9: 695-702, 2000.

18. Fiot E, Zenaty D, Boizeau P, Haigneré J, Dos Santos S and Léger J; French Turner Syndrome Study Group: X-chromosome gene dosage as a determinant of impaired pre and postnatal growth and adult height in Turner syndrome. Eur J Endocrinol 174: 281-288, 2016.

19. Zinn AR, Tonk VS, Chen Z, Flejter WL, Gardner HA, Guerra R, Kushner H, Schwartz S, Sybert VP, Van Dyke DL and Ross JL: Evidence for a Turner syndrome locus or loci at Xp11.2-p22.1. Am J Hum Genet 63: 1757-1766, 1998.

20. Tukiainen T, Pirinen M, Sarin AP, Ladenvall C, Kettunen J, Lehtimäki T, Lokki ML, Perola M, Sinisalo J, Vlachopoulou E, et al: Chromosome X-wide association study identifies Loci for fasting insulin and height and evidence for incomplete dosage compensation. PLoS Genet 10: e1004127, 2014.

21. Seo GH, Kang E, Cho JH, Lee BH, Choi JH, Kim GH, Seo EJ and Yoo HW: Turner syndrome presented with tall stature due to overdosage of the SHOX gene. Ann Pediatr Endocrinol Metab 20: 110-113, 2015.

22. del Rey G, Jasper H, Bengolea SV, Boywitt A, De Bellis R and Heinrich JJ: Trisomy of the short stature homeobox-containing gene (SHOX) due to duplication/deletion of the X chomosome: Clinical implications on the stature. Horm Res Paediatr 74 : 297-304, 2010.

23. Dalton P, Coppin B, James R, Skuse D and Jacobs P: Three patients with a 45,X/46,X,psu $\operatorname{dic}(\mathrm{Xp})$ karyotype. J Med Genet 35: 519-524, 1998. 